\title{
Observation of electronic energy bands in argon clusters
}

\author{
Marko Förstel,,${ }^{1,2}$ Melanie Mucke, ${ }^{1}$ Tiberiu Arion, ${ }^{1}$ Toralf Lischke, ${ }^{1}$ Silko Barth, ${ }^{1}$ Volker Ulrich, ${ }^{1}$ Gunnar Öhrwall,, 3 \\ Olle Björneholm, ${ }^{3}$ Uwe Hergenhahn, ${ }^{1, \dagger}$ and Alex M. Bradshaw ${ }^{1,4}$ \\ ${ }^{1}$ Max-Planck-Institut für Plasmaphysik, EURATOM Association, Boltzmannstr. 2, 85748 Garching, Germany \\ ${ }^{2}$ Max-Planck-Institut für Kernphysik, Saupfercheckweg 1, 69117 Heidelberg, Germany \\ ${ }^{3}$ Uppsala University, Division of Molecular and Condensed Matter Physics, Department of Physics and Astronomy, \\ P.O. Box 516, SE-75120 Uppsala, Sweden \\ ${ }^{4}$ Fritz-Haber-Institut der Max-Planck-Gesellschaft, Faradayweg 4-6, 14195 Berlin, Germany
}

(Received 3 September 2010; published 28 September 2010)

\begin{abstract}
The $3 p$ valence region of argon clusters has been investigated with photoemission near the photoionization threshold. A strong feature between 14.6 and $15.3 \mathrm{eV}$ binding energy shows a photon-energy dependence indicative of electronic-energy band dispersion. A similar feature at approximately the same binding energy and with a similar dispersion occurs in photoemission spectra of both the ordered and disordered solids. The effect is already fully developed for scaling-law mean cluster sizes $\langle N\rangle$ of approximately 200 atoms, thus showing an early onset of bulklike electronic properties.
\end{abstract}

DOI: 10.1103/PhysRevB.82.125450

PACS number(s): 61.46.Bc, 71.20.Ps, 36.40.Cg

\section{INTRODUCTION}

The accepted view in nanoscience is that the very small pieces of matter termed "clusters" bridge the gap between molecules and solids and may even have specific properties of their own. It has been difficult, however, to establish the transition from molecular properties to bulklike behavior as a function of increasing cluster size. How do volume properties evolve as a function of size? When does a cluster become essentially a small piece of the "bulk"? The sizedependent electronic structure of metal clusters has been investigated with photoelectron spectroscopy. Kaiser and Rademann ${ }^{1}$ showed that the valence bandwidth of mercury clusters $\operatorname{Hg}_{N}(N=2-109)$ increases as expected with cluster size; for a cluster with $N=109$ the bandwidth is already $80 \%$ of the bulk value. Later studies on, for example, alkali metals, ${ }^{2}$ aluminum, ${ }^{3}$ and coinage metals ${ }^{4}$ have been interpreted in terms of the jellium model and show direct evidence for the closing of electronic shells. Interestingly, new spectral features appear for $N>100$ in $\mathrm{Al}$ clusters, ${ }^{3}$ which the authors suggest may be due to "lattice-based structures," i.e., those corresponding to the bulk. In the case of weakly bound, van der Waals clusters, fluorescence spectra have shown that the intensity ratio of surface-to-volume excitons in krypton ${ }^{5}$ and $\operatorname{argon}^{6}$ clusters changes as a function of size. In $\mathrm{Kr}$ clusters bulk excitons start to appear above $N=200$. Similarly, recent high-resolution core and valence photoelectron studies of rare-gas clusters have allowed surface and bulk contributions to the spectra to be distinguished. ${ }^{7-9} \mathrm{On}$ the basis of the valence band width and the surface vs bulk contribution as a function of Xe cluster size Rolles et al. ${ }^{8}$ conclude that bulklike electronic band formation occurs for a cluster mean size $\langle N\rangle$ above 500 . The observation of bulklike electronic bands would imply that the long-range order in the cluster is sufficiently great that the electronic states are defined not only by their energy but also by their crystal momentum. So far, there has been no direct evidence for such band dispersions. Since a cluster in a beam experiment is not fixed in space, energy band dispersion in photoemission would normally be difficult to observe. For oriented single crystals its signature is the observation of photon energydependent or angle-dependent shifts of features in the spectrum caused by momentum-conserving optical transitions. ${ }^{10}$ In this paper we report a feature in the photoemission spectra of argon clusters at $\sim 15 \mathrm{eV}$ binding energy which, despite this lack of orientation, shows a strong dispersion effect. The feature reaches full intensity and full dispersion width at mean cluster sizes of 150-200 atoms, indicating that bulklike electronic properties have already developed in this range.

Outer valence photoemission spectra of rare-gas atoms are characterized by a sharp doublet produced by spin-orbit splitting of the $n p$ levels. In contrast, the corresponding spectra of rare-gas clusters show broad, partly structured features containing contributions from both the bulk and the surface..$^{7-9,11-13}$ Only in the case of krypton and xenon is the spin-orbit splitting readily apparent. For argon clusters with $\langle N\rangle=250$ Rolles et al. ${ }^{8}$ have tentatively fitted the profile of the $1.5 \mathrm{eV}$ broad $3 p$-derived feature by assuming that there is only one contribution each from surface and bulk atoms and that the $3 p_{3 / 2}$ level is split into its magnetic sublevels $m_{j}$ $= \pm 3 / 2, \pm 1 / 2$ due to the interaction between neighboring atoms (often referred to as a crystal-field splitting). ${ }^{14-16}$ Thus, in the $1.5 \mathrm{eV}$ broad $3 p$-derived feature six component peaks are expected. Photoelectron spectra of polycrystalline argon films were measured by Schwentner et al. ${ }^{17}$ in the photon-energy range $14-24 \mathrm{eV}$, revealing a valence bandwidth of about $1.8 \mathrm{eV}$. Kassühlke et al. ${ }^{18}$ obtained similar results on $\operatorname{Ar}(111)$ single-crystal films grown epitaxially on $\mathrm{Ru}(001)$; they observed a somewhat broader valence-band and strong dispersion effects. Since these latter data are particularly relevant for the present investigation they are discussed in more detail below.

\section{EXPERIMENTAL}

Photoelectron spectra of Ar clusters were measured at the third generation synchrotron radiation source BESSY II. Clusters are produced by expansion of Ar gas through a liq- 
uid nitrogen-cooled nozzle into an expansion chamber, which is separated from the main interaction chamber by a conical skimmer. ${ }^{19}$ Knowing the nozzle temperature, its geometry and the stagnation pressure the mean size of the clusters $\langle N\rangle$ can be estimated from empirically derived scaling laws, which are used here in a formulation due to Hagena. ${ }^{20}$ High-resolution spectra were recorded with a hemispherical electron analyzer (Scienta) on the UE112/lowE PGMa beamline. Horizontally, linearly polarized radiation was used and the electron-energy analyzer was placed in the dipole plane under the magic angle of $54.7^{\circ}$. Details of this experiment and the data analysis have been given in Ref. 9 (see also Ref. 21). For a cluster size-dependent study, ${ }^{22}$ we have used a newly commissioned apparatus with a horizontal cluster jet and a vertical magnetic bottle-type ${ }^{23}$ electron spectrometer. It was placed at the TGM4 beamline in single bunch mode of operation. As the resolving power of the magnetic bottle spectrometer is $E / \Delta E \approx 20$, these spectra are referred to below as being of "low resolution." An alternative, and probably more accurate, determination of cluster size was made by recording the $\operatorname{Ar} 3 s$ bulk/surface peak intensity ratio ${ }^{9,12}$ with the Scienta analyzer. Spectra shown vs binding energy were calibrated to the ionization energies of the atomic $3 p$ levels, namely, 15.760 and $15.937 \mathrm{eV}^{24}$

\section{RESULTS}

The argon $3 p$ valence region was measured with high resolution for a mean cluster size of $\langle N\rangle=1670$ from the threshold up to a photon energy of $28 \mathrm{eV}$ in steps of $0.2 \mathrm{eV}$. For most energies the spectra are very similar to previous results (e.g., compare to Ref. 8, $h v=26 \mathrm{eV}$ for $\langle N\rangle=250$ and see Ref. 9). In a narrow range of parameter space just above threshold, however, there is an important difference, as shown in Fig. 1. In the photon-energy range 15.5-17.5 eV a very strong feature at $\approx 15 \mathrm{eV}$ binding energy with a halfwidth of about $0.25 \mathrm{eV}$ is visible. This is superimposed on the $1.5 \mathrm{eV}$ broad cluster band on the low binding energy side of the atomic lines. In fact, this feature not only dominates the spectrum but also shifts smoothly and continuously by about $0.7 \mathrm{eV}$ in binding energy from 14.6 to $15.3 \mathrm{eV}$ over this photon-energy range. Figure 1 shows this sequence of spectra in steps of $0.2 \mathrm{eV}$ photon energy from $h v=15.5$ to $18.3 \mathrm{eV}$. Above $h v=17.7 \mathrm{eV}$ the spectra do not change essentially (at least up to $h v=28 \mathrm{eV}$ as measured here) and are characterized by a small feature at a constant binding energy of $15.5 \mathrm{eV}$, as in previous work. The dispersionlike behavior of the feature at $\approx 15 \mathrm{eV}$ binding energy is seen more clearly in a contour plot of intensity vs binding energy and photon energy in Fig. 2, where the peak maxima from Fig. 1 are marked with black dots. Concerning the spectra at $h v=15.5$ and $15.7 \mathrm{eV}$ we must remember that the feature corresponds to a kinetic energy of less than $1 \mathrm{eV}$, for which the transmission of the analyzer is very low which, in turn, makes it hard to differentiate between spectral features and noise.

Also shown schematically in Fig. 2 are the corresponding photoemission data from Kassühlke et al. ${ }^{18}$ for (111) singlecrystal bulk argon recorded in normal emission. They show only two features in the binding energy range $12.5-15.0 \mathrm{eV}$,

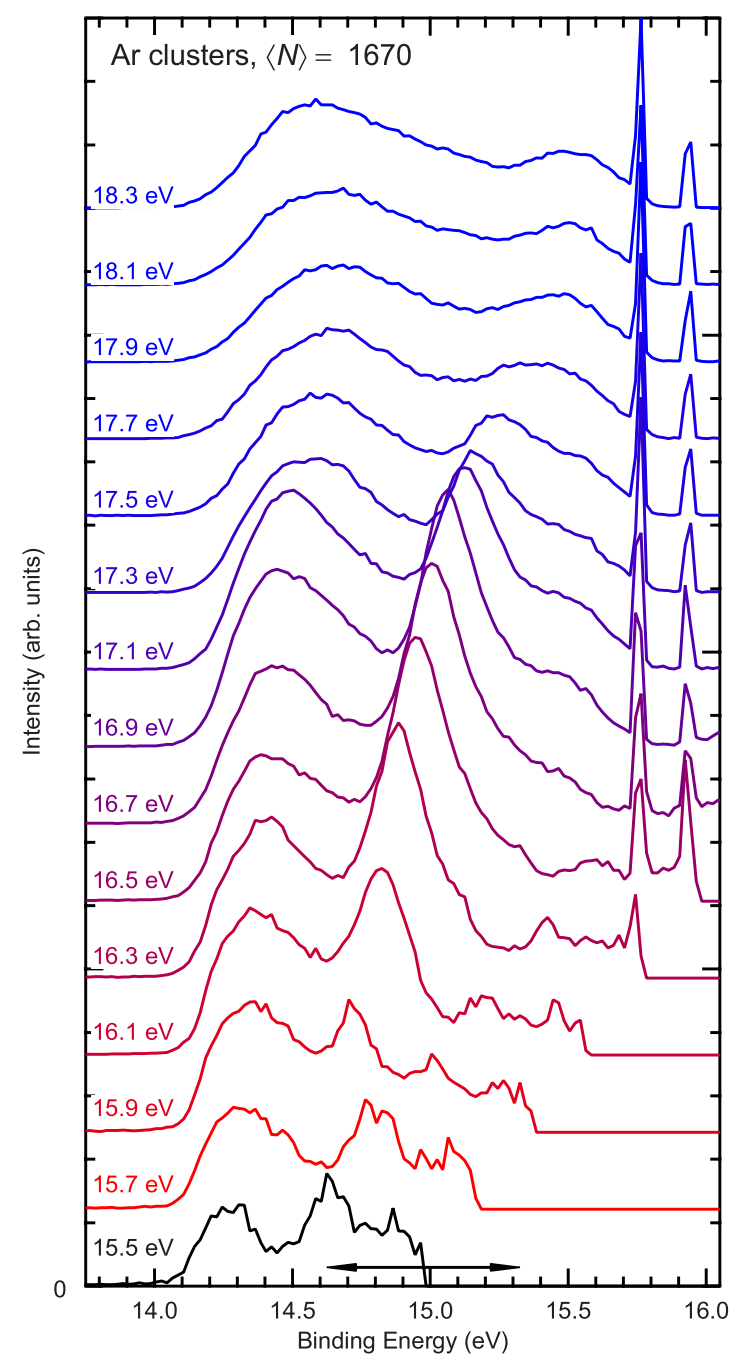

FIG. 1. (Color online) High-resolution $3 p$ (valence band) photoelectron spectra of Ar clusters at photon energies from 15.5 to $18.3 \mathrm{eV}$. The mean cluster size (from the scaling laws) is $\langle N\rangle$ $=1670$. Data recorded with the Scienta analyzer. The sharp, spinorbit split atomic lines at 15.76 and $15.94 \mathrm{eV}$ binding energy are below the usable energy range of the analyzer at $h v=16.1 \mathrm{eV}$. A horizontal arrow marks the extent of the observed dispersion.

one of which-like the feature in the cluster datadominates the spectra between 16 and $18 \mathrm{eV}$ photon energy and also shifts to higher binding energy by about $0.6 \mathrm{eV}$ (dashed line marked " 1 "). In fact, the slope is approximately the same as the corresponding feature from the cluster, although the latter is not as straight. The line marked " 1 " and our feature are separated by about $0.5 \mathrm{eV}$ in binding energy, which is surprisingly small considering the different "substrate" and the resulting differences in final-state effects. The other, weaker feature in the data of Kassühlke et al. (dashed line "2") shifts slightly to lower binding energy with the slope increasing at higher photon energies (not shown). The total shift is about $1.5 \mathrm{eV}$. Interestingly, the strong feature (line 1) is also clearly present in the early photoemission data of Schwentner et al., ${ }^{17}$ whose argon samples were not ordered. We note the identical photon-energy range, the very similar binding-energy shift and the high intensity of the 


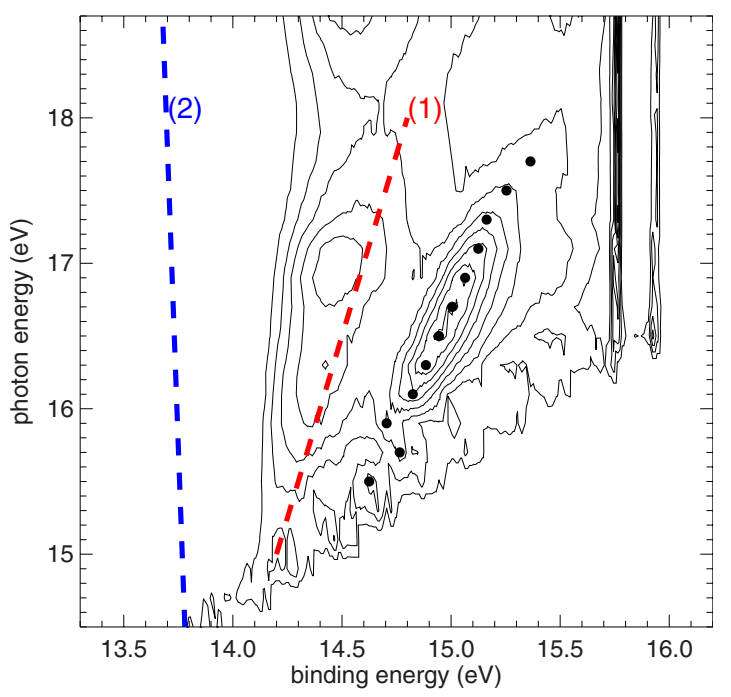

FIG. 2. (Color online) Contour plot showing high-resolution $3 p$ photoelectron spectra of Ar clusters with mean size $\langle N\rangle=1670$ as a function of both photon energy and binding energy. The peak maxima of the $\approx 15 \mathrm{eV}$ feature showing dispersion are indicated by black dots. High-resolution data recorded with the Scienta analyzer. The lines designated 1 and 2 derive from the photoemission data for $\operatorname{Ar}(111)$ measured by Kassühlke et al. (Ref. 18). See text.

$\approx 15 \mathrm{eV}$ feature in the single crystal, polycrystalline film, and cluster data (in all three cases the feature dominates the spectra). We therefore conclude that the same effect is occurring in the cluster as in the bulk and may be attributed to energy band dispersion. In other words, there is direct evidence that at $\langle N\rangle=1670$ the clusters have bulklike electronic properties.

In order to investigate whether there is a threshold for the observation of this effect, we have recorded data at lower resolution using the magnetic bottle analyzer for several smaller cluster sizes. Data sets analogous to Fig. 2 were taken for $\langle N\rangle=24,41,96$, and 191. A full account of these experiments will be given elsewhere, ${ }^{22}$ but here we briefly summarize the main findings. The two lowest cluster sizes give rise to plots resembling Fig. 2, but with a substantially reduced energy shift of the $\approx 15 \mathrm{eV}$ feature. Moreover, it is less intense relative to the background from the cluster valence band. Three representative spectra for $\langle N\rangle=41$, analogous to Fig. 1, are shown in Fig. 3. At $\langle N\rangle=96$ and 191 the shift approaches the value of $0.7 \mathrm{eV}$ shown in Fig. 2 for $\langle N\rangle=1670$. In particular, for $\langle N\rangle=191$ the data resemble closely those of Fig. 2, in that the peak-to-background intensity ratio and the slope photon energy/binding energy match closely the data for the larger cluster size. It therefore appears that the effect is fully developed for $\langle N\rangle=200$, and that the threshold is approximately $\langle N\rangle=100$. We suggest that the similar, but much weaker effect observed at very small values of $\langle N\rangle$ is due to the presence of larger clusters resulting from the broad size distribution. In addition, as a function of mean cluster size, the overall width of the $3 p$ valence band increases from about $1.25 \mathrm{eV}$ for $\langle N\rangle=24$ to about $1.5 \mathrm{eV}$ for $\langle N\rangle=191$. As noted above, in the photoemission spectra of bulk, polycrystalline argon the width of the valence-band emission is about $1.8 \mathrm{eV}^{17}$

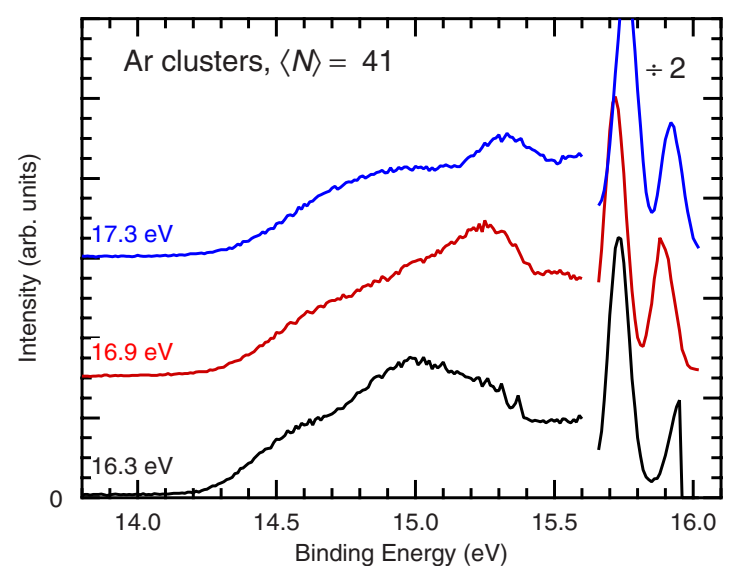

FIG. 3. (Color online) Low-resolution $3 p$ (valence-band) photoelectron spectra of Ar clusters at different photon energies recorded with the magnetic bottle analyzer. The mean cluster size is $\langle N\rangle$ $=41$.

Doubt must remain as to the cluster-size determination using the scaling laws. ${ }^{20}$ An alternative, and possibly more accurate measure of mean cluster size is provided by the bulk-to-surface area ratio of a core or inner valence photoelectron line. ${ }^{12}$ In an independent experiment, we have therefore determined this quantity from $3 s$ photoelectron spectra taken under expansion conditions relevant to this work. In agreement with our earlier study ${ }^{9}$ we find that the mean sizes derived from the photoemission are larger than those from the scaling laws. The agreement is better for larger cluster sizes. For $\langle N\rangle=40$ photoemission results in a mean size of 160 , for $\langle N\rangle=150$ the corresponding figure is $300 .{ }^{22}$ The threshold for the establishment of bulklike electronic bands would then lie at about $N_{\mathrm{ph}}=230$, where the index "ph" denotes "photoemission."

\section{DISCUSSION}

Are there conceivable explanations for the effect other than dispersion? One possible explanation could be a depth dependence of the binding energy of the argon levels as a result of either initial-state effects or screening in the final state. In general, the mean free path of electrons in solids, and thus the probing depth in photoelectron spectroscopy, can change strongly at very low kinetic energies. This can be ruled out, however, for the argon clusters studied here. First, the threshold for electron-electron scattering in solid argon has been measured by Schwentner ${ }^{25}$ to be about $12 \mathrm{eV}$ kinetic energy relative to the top of the valence band, i.e., about twice the energy of the first exciton. In the kineticenergy range of relevance here, namely, $1.2-2.2 \mathrm{eV}$, the photoelectrons thus have a mean free path of at least a hundred nanometers. This means that even those emitted inside the cluster are essentially unattenuated. Second, even if there were a meaningful difference in photoemission binding energy between the surface and deeper layers, clusters in this size range consist of only three to four atomic layers! For the effect in question we need to explain a smooth, continuous shift over a binding-energy range which is three times the 
halfwidth of the feature. This latter fact also argues against other "final-state" explanations associated with earlier observations on very small clusters. For example, small ionic raregas clusters exhibit stronger bonding than their neutral counterparts, and the formation of ionic cores (dimers, trimers, etc.) in the ionization of clusters has been discussed. ${ }^{26,27}$ Attempts to model the binding-energy spectrum from properties of these ionic cores were, however, less successful (see discussion in Ref. 9). We therefore believe that such finalstate effects occur on longer time scales, as seen, e.g., in ion spectroscopy. ${ }^{26,27}$

Since band-structure effects are clearly observed in the present work, it is interesting to look at the situation with regard to electronic-structure calculations for bulk argon. The calculated GW band structure of GalamićMulaomerović and Patterson ${ }^{28}$ published some 40 years after the first rare-gas band-structure calculations, ${ }^{29,30}$ seems to reproduce quantitatively (for the first time) most of the experimental data for the bulk: single-particle excitation energies, valence bandwidth and electron affinity. As described above, some of the normal emission data of Kassühlke et al. ${ }^{18}$ for 〈111) single-crystal films have been included in Fig. 2 (dashed lines marked 1 and 2). We tentatively assign the band (1) to the $3 p$-derived band along $\Gamma_{15}-\mathrm{L}_{2}$, although the extent of the measured dispersion $(\approx 0.6 \mathrm{eV})$ is not as large as the bandwidth $(1.83 \mathrm{eV})$ in the calculation of Ref. 28 . Similarly, the feature shown schematically by the dashed line 2 may be due the $3 p$-derived $\Gamma_{15}-\mathrm{L}_{3}$ band. Thus, our interpretation of the bands 1 and 2 from the single-crystal data ${ }^{18}$ locates the $\Gamma_{15}$ point (corresponding to the top of the valence band) at about $14 \mathrm{eV}$ binding energy, consistent with the literature value of $13.8 \mathrm{eV}$. The high intensity of band 1 , in particular, between 16 and $18 \mathrm{eV}$, where it dominates the spectrum, is probably a result of strong optical absorption due to interband transitions. The electron energy-loss spectrum of solid argon also shows a prominent feature in this spectral region. ${ }^{31}$

One important question-interesting from the photoemission point of view-is why dispersion is observed in disordered polycrystalline systems. Even if the clusters can be described as very small crystallites with an fcc, or near-fcc structure, ${ }^{32}$ their orientation in the beam is random. One possibility is that the extent of the dispersion of the $3 p$ band along $\Gamma \mathrm{L}$ and its lack of hybridization with other bands is very similar to that along $\Gamma \mathrm{X}$, and probably to other directions of the Brillouin zone, leading to almost isotropic emission, providing that appropriate final-state bands are available. (See Ref. 10, Chap. 7.) Alternatively, and perhaps more likely, if the transition is restricted to a narrow range of directions in the Brillouin zone, those clusters with approximately the correct orientation for observing emission from that direction will produce the effect, particularly if the matrix element is large.

The cluster-size distribution is relatively broad, ${ }^{33,34}$ but on the assumption that the particles have the near-fcc, icosahedral structure $N=230$ corresponds to somewhat more than three complete shells, which occurs at $N=147$. Farges et al. ${ }^{35}$ interpreted their electron-diffraction data for $\langle N\rangle=50-750$ in terms of an icosahedral structure, but later studies ${ }^{32,36}$ have not necessarily come to the same conclusion and there re- mains some doubt as to the structure of the clusters in the size range studied here. Despite these uncertainties, the present data show that a dispersion effect indicative of bulklike, and probably of fcc-like, behavior is visible for cluster sizes $N_{\mathrm{ph}} \approx 230$. This "early" transition from molecular to bulklike properties is perhaps at first sight surprising but does have a simple explanation. Let us assume the presence of small crystallites with a bulk fcc structure and a diameter of about six or seven atoms, corresponding to just over three complete shells. If we consider the bonding of, say, the $3 p_{x}$ orbitals in a one-dimensional row of seven argon atoms, then in a linear combination of atomic orbitals, or simple tightbinding model, seven discrete levels would be created with a separation of about $0.7 \mathrm{eV}$, (the measured dispersion) between the most bonding molecular orbital and the most antibonding molecular orbital. The observation of a smooth continuous shift of the strong $\approx 15 \mathrm{eV}$ feature in the present experiment then implies that the intrinsic broadening mechanisms (and/or the instrumental resolution) give rise to an observed linewidth which is greater than the mean separation between the discrete levels. Continuing in this simple picture, the separation is expected to be approximately $0.1 \mathrm{eV}$, but already smeared out because of the cluster-size distribution and the effect of three dimensions. On the other hand, the linewidth of the $\approx 15 \mathrm{eV}$ feature is about $0.25 \mathrm{eV}$, i.e., considerably higher than that due to any remaining discrete levels. Instrumental linewidth clearly does not play a role, since there is essentially no difference between the high- and low-resolution data sets as far as the $\approx 15 \mathrm{eV}$ feature is concerned. Concerning the bonding, we should note in passing that the transition to "bandlike" behavior, although of course interesting in itself, is not accompanied by any fundamental change in the electronic structure. The decisive mechanism giving rise to bonding in the dimer, in clusters and in the solid is the van der Waals interaction, not "chemical" bonding. In terms of classical electrodynamics the former is due to the motion of the electrons on different atoms giving rise to fluctuating multipoles and a resultant attractive interaction (as in the long-range part of the Lennard-Jones pair potential). Bonding, or band formation, via the overlap of electronic wave functions is for the rare gases always a repulsive interaction.

Although the present work has been able to demonstrate the transition from cluster to bulk properties, the broad distribution, and the rather approximate determination of the mean cluster size still represent a handicap. Possible future experiments could utilize photoelectron-cluster ion coincidence spectroscopy, e.g., Refs. 1 and 37, although in the case of rare-gas clusters evaporation of neutrals in the final state would have to be quantified. ${ }^{34}$

\section{CONCLUSIONS}

Because of the difficulty of size selecting (in sufficient numbers), isolating and orienting clusters, most of the usual techniques for determining structure and electronic properties cannot be used in their investigation. For this reason, there is, in general, only indirect information on the two key questions in cluster research. How do volume properties 
evolve as a function of size? When does a cluster essentially become a small piece of the bulk? In the present investigation we observe a strong feature in the valence-band region of the photoemission spectrum of argon clusters, the binding energy of which changes continuously with the photon energy. The feature is sharp with a full width at half maximum of $\sim 0.25 \mathrm{eV}$, but the almost linear change in binding energy is about a factor three greater, namely, $0.7 \mathrm{eV}$, which is an indication of electronic band dispersion. The photon-energy range over which it is observed is $15.5-17.5 \mathrm{eV}$. This sharp feature is unique in the photoemission spectrum and is only to be found in a very small region of parameter space. The feature displays the full extent of the binding-energy change with coverage after a mean cluster size of 200 atoms (estimated with the scaling laws) has been reached. The threshold for the appearance of this bandlike behavior is approximately 100 atoms. Using alternatively a photoemission experiment to calibrate the cluster size we arrive at a value of $N_{\mathrm{ph}}$ $\approx 230$ atoms for this threshold. A very similar band has been observed in almost exactly the same photon-energy and binding-energy ranges for both polycrystalline argon films and (111)-oriented argon single crystals. The most reason- able assignment for this feature is electronic-energy band dispersion associated with interband transitions in singlecrystal (fcc) argon. This is an unexpected result for a cluster beam with random orientation of the crystallites but is explicable with a conventional photoemission model. What is the significance of these data? First, we have been able to determine at which point bulklike electronic-band formation begins. Second, the result is surprising because the transition is not expected to occur at such a small cluster size. We offer a very simple tight-binding model to explain the data.

\section{ACKNOWLEDGMENTS}

We thank P. Feulner, K. Horn, D. Menzel, A. Nilsson, M. Scheffler, A. Stampfl, and G. P. Williams for useful discussions. Moreover, we acknowledge financial support from the Deutsche Forschungsgemeinschaft, the Advanced Study Group of the Max-Planck-Society, the Fonds der chemischen Industrie, the Swedish Research Council (VR), and the Seventh Framework Programme of the European Community (FP7/2007-2013; grant Agreement No. 226716).
*Present address: MAX-lab, Lund University, P.O. Box 118, SE22100 Lund, Sweden.

${ }^{\dagger}$ Mail address: c/o Helmholtz-Zentrum Berlin, Albert-Einstein-Str. 15, 12489 Berlin, Germany; uwe.hergenhahn@ipp.mpg.de

${ }^{1}$ B. Kaiser and K. Rademann, Phys. Rev. Lett. 69, 3204 (1992).

${ }^{2}$ G. Wrigge, M. Astruc Hoffmann, and B. v. Issendorff, Phys. Rev. A 65, 063201 (2002).

${ }^{3}$ X. Li, H. Wu, X.-B. Wang, and L.-S. Wang, Phys. Rev. Lett. 81, 1909 (1998).

${ }^{4}$ H. Handschuh, C. Y. Cha, P. S. Bechthold, G. Ganteför, and W. Eberhardt, J. Chem. Phys. 102, 6406 (1995).

${ }^{5}$ J. Stapelfeldt, J. Wörmer, and T. Möller, Phys. Rev. Lett. 62, 98 (1989).

${ }^{6}$ J. Wörmer, M. Joppien, G. Zimmerer, and T. Möller, Phys. Rev. Lett. 67, 2053 (1991).

${ }^{7}$ R. Feifel, M. Tchaplyguine, G. Öhrwall, M. Salonen, M. Lundwall, R. R. T. Marinho, M. Gisselbrecht, S. L. Sorensen, A. Naves de Brito, L. Karlsson, N. Mårtensson, S. Svensson, and O. Björneholm, Eur. Phys. J. D 30, 343 (2004).

${ }^{8}$ D. Rolles, H. Zhang, Z. D. Pešić, J. D. Bozek, and N. Berrah, Chem. Phys. Lett. 468, 148 (2009).

${ }^{9}$ U. Hergenhahn, S. Barth, V. Ulrich, M. Mucke, S. Joshi, T. Lischke, A. Lindblad, T. Rander, G. Öhrwall, and O. Björneholm, Phys. Rev. B 79, 155448 (2009).

${ }^{10} \mathrm{~S}$. Hüfner, Photoelectron Spectroscopy, 3rd ed. (Springer-Verlag, Berlin, 2003).

${ }^{11}$ U. Hergenhahn, A. Kolmakov, M. Riedler, A. R. B. de Castro, O. Löfken, and T. Möller, Chem. Phys. Lett. 351, 235 (2002).

${ }^{12}$ M. Tchaplyguine, R. R. Marinho, M. Gisselbrecht, J. Schulz, N. Mårtensson, S. L. Sorensen, A. Naves de Brito, R. Feifel, G. Öhrwall, M. Lundwall, S. Svensson, and O. Björneholm, J. Chem. Phys. 120, 345 (2004).

${ }^{13}$ T. Hatsui, H. Setoyama, N. Kosugi, B. Wassermann, I. L.
Bradeanu, and E. Rühl, J. Chem. Phys. 123, 154304 (2005).

${ }^{14}$ K. Horn, M. Scheffler, and A. M. Bradshaw, Phys. Rev. Lett. 41, 822 (1978).

${ }^{15}$ K. Jacobi, Phys. Rev. B 38, 5869 (1988).

${ }^{16}$ K. Horn, C. Mariani, and L. Cramer, Surf. Sci. 117, 376 (1982).

${ }^{17}$ N. Schwentner, F.-J. Himpsel, V. Saile, M. Skibowski, W. Steinmann, and E. E. Koch, Phys. Rev. Lett. 34, 528 (1975).

${ }^{18}$ B. Kassühlke, P. Feulner, and D. Menzel (private communication); see B. Kassühlke, Ph.D. thesis, Technical University of Munich, 1999 (Herbert Utz Verlag, Munich, 1998).

${ }^{19}$ S. P. Marburger, O. Kugeler, and U. Hergenhahn, in Synchrotron Radiation Instrumentation: Eighth International Conference, edited by T. Warwick, J. Arthur, H. A. Padmore, and J. Stöhr (AIP, San Francisco, 2003), Vol. 705, p. 1114.

${ }^{20}$ O. F. Hagena, Rev. Sci. Instrum. 63, 2374 (1992).

${ }^{21}$ Table I in Ref. 9 contains minor inaccuracies in the relevant column labelled "Fig. 3." It should read $p=1630$ mbar (not 1500), $T=95 \mathrm{~K}$ (not 89), which results in the cluster size of 1670 given here.

${ }^{22}$ M. Förstel, M. Mucke, T. Arion, T. Lischke, S. Barth, V. Ulrich, G. Öhrwall, O. Björneholm, U. Hergenhahn, and A. M. Bradshaw, J. Electron Spectrosc. Relat. Phenom. (to be published).

${ }^{23}$ P. Kruit and F. H. Read, J. Phys. E 16, 313 (1983).

${ }^{24}$ I. Velchev, W. Hogervorst, and W. Ubachs, J. Phys. B 32, L511 (1999).

${ }^{25}$ N. Schwentner, Phys. Rev. B 14, 5490 (1976).

${ }^{26}$ G. Ganteför, G. Broker, E. Holub-Krappe, and A. Ding, J. Chem. Phys. 91, 7972 (1989).

${ }^{27}$ W. Kamke, J. de Vries, J. Krauss, E. Kaiser, B. Kamke, and I. V. Hertel, Z. Phys. D: At., Mol. Clusters 14, 339 (1989).

${ }^{28}$ S. Galamić-Mulaomerović and C. H. Patterson, Phys. Rev. B 71, 195103 (2005)

${ }^{29}$ L. F. Mattheiss, Phys. Rev. 133, A1399 (1964). 
${ }^{30}$ U. Rössler, Phys. Status Solidi B 42, 345 (1970).

${ }^{31}$ L. Schmidt, Phys. Lett. A 36, 87 (1971).

${ }^{32}$ S. Kakar, O. Björneholm, J. Weigelt, A. R. B. de Castro, L. Tröger, R. Frahm, T. Möller, A. Knop, and E. Rühl, Phys. Rev. Lett. 78, 1675 (1997).

${ }^{33}$ R. Karnbach, M. Joppien, J. Stapelfeldt, J. Wörmer, and T. Möller, Rev. Sci. Instrum. 64, 2838 (1993).
${ }^{34}$ S. Schütte and U. Buck, Int. J. Mass. Spectrom. 220, 183 (2002).

${ }^{35}$ J. Farges, M. F. de Feraudy, B. Raoult, and G. Torchet, J. Chem. Phys. 84, 3491 (1986).

${ }^{36}$ B. W. van de Waal, G. Torchet, and M.-F. de Feraudy, Chem. Phys. Lett. 331, 57 (2000).

${ }^{37}$ K. Rademann, T. Rech, B. Kaiser, U. Even, and F. Hensel, Rev. Sci. Instrum. 62, 1932 (1991). 